\title{
The impact of complete blood count-derived inflammatory markers on survival in patients with localized renal cell cancer treated with partial or radical nephrectomy - a single center retrospective study
}

Łukasz Zapała ( $\nabla$ zapala.lukasz@gmail.com )

Medical University of Warsaw https://orcid.org/0000-0001-7340-280X

Aleksander Ślusarczyk

Clinic of General, Oncological and Functional Urology, Medical University of Warsaw

Karolina Garbas

Clinic of General, Oncological and Functional Urology, Medical University of Warsaw

Łukasz Mielczarek

Clinic of General, Oncological and Functional Urology, Medical University of Warsaw

Cezary Ślusarczyk

Clinic of General, Oncological and Functional Urology, Medical University of Warsaw

Piotr Zapała

Clinic of General, Functional and Oncological Urology, Medical University of Warsaw

Andrzej Wróbel

Second Department of Gynecology, Medical University of Lublin

Piotr Radziszewski

Clinic of General, Functional and Oncological Urology, Medical University of Warsaw

\section{Research}

Keywords: renal cell carcinoma, biomarkers, survival analysis, systemic inflammatory markers

Posted Date: September 30th, 2021

DOI: https://doi.org/10.21203/rs.3.rs-940202/v1

License: (c) (i) This work is licensed under a Creative Commons Attribution 4.0 International License. Read Full License 


\section{Abstract}

Backround

Several hematological markers of systemic inflammation were reported as prognostic in renal cell carcinoma (RCC). We aimed to re-evaluate the prognostic significance of clinicopathologic features and compare the predictive value of different inflammatory markers in RCC.

Methods

Four hundred ninety-five patients treated with nephrectomy for primary localized or locally advanced RCC were included in the retrospective analysis. The median follow-up was 48 months.

Results

Patients with higher neutrophil/lymphocyte ratio (NLR), platelet/lymphocyte ratio (PLR), systemic inflammatory response index (SIRI), systemic immune-inflammation index (SII), neutrophil/erythrocyte ratio (NER), derived neutrophil/lymphocyte ratio (dNLR), and lower lymphocyte/monocyte ratio (LMR) and hemoglobin/platelet ratio (HPR) had worse cancer-specific survival (CSS). In the multivariate analysis tumour stage, grade, age and high SIRI constituted independent factors predicting CSS. The model including SIRI values achieved C-index 0.903. Alternative multivariate models that included SII and NLR were characterized by comparable C-indexes i.e. 0.902 and 0.890, respectively.

Different inflammatory markers could not be utilized in a single model, as they show a strong correlation with one other (SII, SIRI and NLR), and seem to provide similar prognostic information. Tumour grade and diameter were independent predictors for recurrence-free survival, whereas age, tumour grade and high NER (or high SIRI/ SII in alternative models) were prognostic for overall survival.

Conclusions

Markers of systemic inflammation might provide additional prognostic information (especially SIRI, SII, NLR and NER) and further increase the predictive accuracy of already available models in localized and locally advanced renal cell carcinoma. Clinicopathological features (stage, grade and age) remain the most important prognostic factors for oncological outcomes in RCC patients treated with nephrectomy. For the first time, we show the prognostic value of neutrophil-to-erythrocyte ratio, which constitutes an independent risk factor of overall survival.

Trial registration

not applicable

\section{Backround}

Localized or locally advanced renal cell carcinoma (RCC) remains the disease that is treated surgically with curative intent [1]. The main concern, though, is connected with a significant rate of late recurrence, reaching in some publications up to $12 \%$ [2]. Specific markers are under investigation to be used in prognostic models, as staging and grading appear to be insufficient, and one observes various outcomes in cases of the indistinguishable clinicopathological characters [3]. The preoperative, early assessment may enforce the optimization of personalized therapy and better risk stratification of patients treated surgically [4]. Then, the appropriate grouping of patients is important for the sake of future management techniques (e.g. surgery versus active surveillance), an adequate followup scheme implementation, and selection of optimal candidates for potential adjuvant treatment inside clinical trials [5]. 
As a great variety of studies focused on hematological markers, they are used more and more frequently for prognostic purposes $[6,7]$. The idea of their clinical implementation comes from the observations on specific interactions within the tumor microenvironment and immune system effector cells [8]. Peripheral blood neutrophils, lymphocytes, monocytes and platelets that are involved in systemic inflammatory reactions are available from routine preoperative blood tests [9]. Within the tumor microenvironment, certain differences in the subpopulations of leukocytes lie at the root of host immunity and reflect tumor aggressiveness [8]. As a consequence, respective peripheral blood count-derived parameters are under investigation for their use in oncology as potential markers, among them most recognizable are the neutrophil-to-lymphocyte ratio (NLR), platelet-to-lymphocyte ratio (PLR), and monocyte-to-lymphocyte ratio (MLR) [6]. Other researchers estimated the role of combinations of the peripheral blood counts' values in a variety of scores, such as the systemic inflammation response index (SIRI) [4], or the systemic immune-inflammation index (SII) [10]. The hope for their future incorporation into everyday practice is based on the fact that the combination of three parameters (e.g. neutrophils, lymphocytes, platelets) better reflects the prognosis and may result in greater accuracy of the respective predictive model [4]. However, the potential utility of the systemic inflammatory markers in the prediction of RCC treatment outcomes is still undetermined. Not to mention the fact that different markers were not simultaneously studied and sufficiently compared in terms of their predictive accuracy. Additionally, the optimal cutoff values of several markers are still a matter of analyses [6]. Worth emphasizing is the fact that these markers are easily obtainable from routine laboratory tests performed in everyday practice, and hence provide an inexpensive and widely available tool for clinicians [9]. The additional use of hemoglobin-to-platelet-ratio (HPR) and neutrophil-to-erythrocyte ratio (NER) represent the novel idea of focusing on red blood cell lineage within anti-cancer response based on the experience coming e.g. from the oncological outcomes of the bladder cancer management described in other papers [11], and in our previous studies [12].

The usefulness of the prognostic models to categorize the cancer progression risk is significantly increased, when the combination of pre-treatment data is engaged [9], while some of the inflammatory markers were found to act as independent prognostic factors also in non-metastatic RCC [7]. Lastly, if novel therapeutics that are targeted to inflammatory pathways are engaged, preoperative values of biomarkers may serve as critical inclusion criteria and become accessory prognostic factors for patients [13].

In the present paper, we aimed to re-evaluate the prognostic significance of clinicopathologic features and compare the predictive value of different inflammatory markers in patients with localized or locally advanced RCC, who received radical or partial nephrectomy.

\section{Methods}

\section{Study population}

This is a retrospective single-tertiary-center study. Medical records of patients treated with partial or radical nephrectomy between 2010 and 2018 were reviewed. Among 778 nephrectomies, 654 were performed because of renal cell carcinoma. Fifty-four patients with distant metastases at the time of surgery and 5 patients with surgery for RCC recurrence (and no data on primary RCC) were excluded from further analysis. For further 6 patients the data were missing and for 90 patients there was no follow-up. Finally, 495 patients with primary localized and locally advanced RCC, who underwent nephrectomy in our center, were included in the analysis.

Information about demographic, clinical and pathological features were collected. None of the patients received additional treatment before radical surgery. Data on the modality of surgery, type of nephrectomy, perioperative complications and the length of hospitalization were gathered, as well. Data extracted from the pathology report 
included tumour stage, grade and histological subtypes. Tumours were graded according to the WHO/ ISUP or Fuhrman classification when adequate. Preoperative laboratory findings were also collected.

Ratio between respective blood cell counts were calculated to produce several inflammatory markers (neutrophil/lymphocyte ratio- NLR, platelet/lymphocyte ratio PLR, lymphocyte/monocyte ratio - LMR, derived neutrophil/lymphocyte ratio- $\mathrm{dNLR}$, hemoglobin/platelet ratio- HPR and neutrophil/erythrocyte ratio- NER). The systemic immune-inflammation index (SII) was calculated according to the following formula: SII = neutrophil $x$ platelet/lymphocyte. The systemic inflammatory response index (SIRI) was calculated as in the formula: SIRI = neutrophil $\times$ monocyte/lymphocyte. Follow-up data included information about recurrence-free survival (RFS), overall survival (OS) and cancer-specific survival (CSS). Recurrence was defined as the detection of a new enhancing lesion in the region of surgery (e.g. surgery bed, ipsilateral adrenal gland) or systemic distant sites during follow-up imaging. Patients were censored at the time of the last follow-up. All patients signed informed consent and the study was conducted under the Ethics committee vote AKBE/72/2021 of the Medical University of Warsaw.

\section{Statistical analysis}

Statistical analysis was performed in SAS software version 9.4. Results are presented as the number and percentage for categorical variables and medians, accompanied by the interquartile range (IQR) for continuous variables. Differences between groups were tested with Fisher's test for categorized variables and Mann-Whitney $U$ test for continuous variables. The differences in the time to the event were analyzed using the Kaplan-Meier method and log-rank test. In univariable analyses Cox proportional hazards were used to identify factors associated with RFS, OS and CSS. Continuous values of inflammatory markers were categorized based on the cutoff determined with receiver operating curve (ROC) and Youden's index analysis. Correlations between variables were checked using the Spearman test. Univariable analyses provided variables for multivariable analyses performed with Cox proportional hazard. Hazard ratio (HR) supplemented with $95 \%$ confidence interval $(95 \% \mathrm{Cl})$ were generated. Several multivariable models were constructed using alternative inflammatory markers, which demonstrated significance in univariable analyses. Inflammatory markers showing strong correlations between each other were not incorporated into a single model. To assess the predictive accuracy for each model the Harell's C-index was calculated. For all statistical analyses, a twosided $p$-value $<0.05$ was considered statistically significant.

\section{Results}

\section{Basic characteristics}

There were 495 consecutive patients enrolled in the study, predominantly male (60\%) (Table 1). The majority of tumors were T1a stage (66\%) and grade 2 (61\%). Pathological examination revealed clear-cell carcinoma in nearly $80 \%$ of cases. Patients were treated surgically with radical intent and, in the majority of cases, nephron-sparing surgeries were performed (68\%). During the median follow-up of 48 months (IQR 30-58) cancer-specific survival reached $95.35 \%$, overall survival $-91.31 \%$, while recurrence-free survival $-89.29 \%$. Baseline patients' characteristics are presented in Table 1. The associations between the values of selected markers and clinicopathologic features were analyzed. Elevated SIRI, SII and NLR were related to advanced staging, larger tumor size, and performance of radical nephrectomy, whereas high SIRI was additionally associated with male gender and tumour grade. The associations in the subgroups with low and high values of inflammatory markers (NLR, SII, SIRI) are summarized in Table 2. 
Table 1

Overall characteristics of the cohort

\begin{tabular}{|c|c|c|c|}
\hline \multicolumn{2}{|l|}{ Characteristics } & \multirow{2}{*}{$\begin{array}{l}\text { No. / Mean } \\
195\end{array}$} & \multirow{2}{*}{$\begin{array}{l}\% / \text { IQR } \\
39.4\end{array}$} \\
\hline Gender & female & & \\
\hline & male & 300 & 60.6 \\
\hline Age & & 63 & $54-70$ \\
\hline BMI & $\mathrm{kg} / \mathrm{m}^{2}$ & 24.7 & $27.8-31.3$ \\
\hline \multirow[t]{6}{*}{ Stage } & $\mathrm{T} 1 \mathrm{a}$ & 327 & 66.06 \\
\hline & $\mathrm{T} 1 \mathrm{~b}$ & 100 & 20.20 \\
\hline & $\mathrm{T} 2 \mathrm{a}$ & 21 & 4.24 \\
\hline & $\mathrm{T} 2 \mathrm{~b}$ & 9 & 1.82 \\
\hline & T3 & 36 & 7.27 \\
\hline & $\mathrm{T} 4$ & 2 & 0.40 \\
\hline \multirow[t]{4}{*}{ Grade } & 1 & 136 & 27.47 \\
\hline & 2 & 305 & 61.62 \\
\hline & 3 & 41 & 8.28 \\
\hline & 4 & 13 & 2.63 \\
\hline Tumour diamater & $\mathrm{cm}$ & 3.7 & $2.5-5.0$ \\
\hline \multirow[t]{4}{*}{ Histology type } & clear cell & 395 & 79.80 \\
\hline & papillary type 1 & 58 & 11.72 \\
\hline & papillary type 2 & 18 & 3.64 \\
\hline & chromofobe & 24 & 4.85 \\
\hline \multirow[t]{2}{*}{ Surgical treatment } & Radical nephrectomy & 156 & 31.52 \\
\hline & NSS & 339 & 68.48 \\
\hline \multicolumn{4}{|l|}{ Outcomes } \\
\hline \multirow[t]{2}{*}{ Recurrence } & No & 442 & 89.29 \\
\hline & Yes & 53 & 10.71 \\
\hline \multirow[t]{2}{*}{ Death } & No & 452 & 91.31 \\
\hline & Yes & 43 & 8.69 \\
\hline \multirow[t]{2}{*}{ Cancer Death } & No & 472 & 95.35 \\
\hline & Yes & 23 & 4.65 \\
\hline Follow-up & months & 48.2 & $30.1-58.1$ \\
\hline Laboratory param & & & \\
\hline
\end{tabular}




\begin{tabular}{|lll|}
\hline Characteristics & No. / Mean & $\% /$ IQR \\
\hline Hemoglobin & 139 & $127-148$ \\
\hline Neutrophils & 4.42 & $3.37-5.73$ \\
\hline Lymphocytes & 1.85 & $1.48-2.38$ \\
\hline Platelets & 225 & $191-280$ \\
\hline Red blood cells & 4.69 & $4.34-4.98$ \\
\hline SIRI & 1.87 & $1.26-2.84$ \\
\hline SII & 506 & $346-807$ \\
\hline
\end{tabular}


Table 2

Association of clinicopathologic features and treatment outcomes with values of inflammatory markers (NLR, SII, SIRI). Note: Number of patients (No.) with percentage (\%) is shown for categorized variables and median with interquartile range (IQR) for continuous variables. Differences were tested with Fisher's test for categorized variables and Mann-

Whitney U test for continuous variables.

\begin{tabular}{|c|c|c|c|c|c|c|c|c|c|c|}
\hline & & $\begin{array}{l}\text { NLR }< \\
2.75\end{array}$ & $\begin{array}{l}\text { NLR } \geq \\
2.76\end{array}$ & & $\begin{array}{l}\text { SIRI } \leq \\
2.25\end{array}$ & $\begin{array}{l}\text { SIRI> } \\
2.25\end{array}$ & & $\begin{array}{l}\text { SII } \leq \\
628\end{array}$ & $\begin{array}{l}\text { SII > } \\
628\end{array}$ & \\
\hline & & $\begin{array}{l}\text { No. (\%) } \\
\text { / } \\
\text { Median } \\
\text { (IQR) }\end{array}$ & $\begin{array}{l}\text { No. (\%) } \\
\text { / } \\
\text { Median } \\
\text { (IQR) }\end{array}$ & $P$ & $\begin{array}{l}\text { No. (\%) } \\
\text { / } \\
\text { Median } \\
\text { (IQR) }\end{array}$ & $\begin{array}{l}\text { No. } \\
\text { (\%)/ } \\
\text { Median } \\
\text { (IQR) }\end{array}$ & $\mathrm{P}$ & $\begin{array}{l}\text { No. (\%) } \\
\text { / } \\
\text { Median } \\
\text { (IQR) }\end{array}$ & $\begin{array}{l}\text { No. } \\
\text { (\%)/ } \\
\text { Median } \\
\text { (IQR) }\end{array}$ & $P$ \\
\hline \multirow[t]{2}{*}{ Gender } & Male & $\begin{array}{l}189 \\
(59)\end{array}$ & $\begin{array}{l}111 \\
(64)\end{array}$ & 0.25 & $\begin{array}{l}173 \\
(55)\end{array}$ & $\begin{array}{l}127 \\
(69)\end{array}$ & 0.002 & $\begin{array}{l}194 \\
(63)\end{array}$ & $\begin{array}{l}106 \\
(56)\end{array}$ & 0.11 \\
\hline & Female & $\begin{array}{l}133 \\
(41)\end{array}$ & $62(36)$ & & $\begin{array}{l}139 \\
(45)\end{array}$ & $56(31)$ & & $\begin{array}{l}112 \\
(37)\end{array}$ & $83(44)$ & \\
\hline Age & & $\begin{array}{l}63 \\
(54- \\
69)\end{array}$ & $\begin{array}{l}63 \\
(55- \\
71)\end{array}$ & 0.23 & $\begin{array}{l}63 \\
(54- \\
69)\end{array}$ & $\begin{array}{l}62 \\
(55- \\
71)\end{array}$ & 0.38 & $\begin{array}{l}63 \\
(55- \\
70)\end{array}$ & $\begin{array}{l}62 \\
(52-69\end{array}$ & 0.37 \\
\hline $\mathrm{BMI}$ & $\mathrm{kg} / \mathrm{m}^{2}$ & $\begin{array}{l}27.8 \\
(24.8- \\
31.2)\end{array}$ & $\begin{array}{l}27.7 \\
(24.6- \\
31.8)\end{array}$ & 0.89 & $\begin{array}{l}27.6 \\
(24.7- \\
31.2)\end{array}$ & $\begin{array}{l}28 \\
(25.2- \\
31.8)\end{array}$ & 0.58 & $\begin{array}{l}27.8 \\
(25.2- \\
31.2)\end{array}$ & $\begin{array}{l}27.3 \\
(24.2- \\
31.8)\end{array}$ & 0.56 \\
\hline \multirow[t]{4}{*}{ Stage } & $\mathrm{T} 1$ & $\begin{array}{l}287 \\
(89)\end{array}$ & $\begin{array}{l}139 \\
(80)\end{array}$ & 0.016 & $\begin{array}{l}283 \\
(91)\end{array}$ & $\begin{array}{l}143 \\
(78)\end{array}$ & 0.001 & $\begin{array}{l}280 \\
(92)\end{array}$ & $\begin{array}{l}146 \\
(77)\end{array}$ & $\begin{array}{l}< \\
0.0001\end{array}$ \\
\hline & $\mathrm{T} 2$ & $15(5)$ & $16(9)$ & & $13(4)$ & $18(10)$ & & $11(4)$ & $20(11)$ & \\
\hline & T3 & $20(6)$ & $16(9)$ & & $16(5)$ & $20(11)$ & & $15(5)$ & $21(11)$ & \\
\hline & $\mathrm{T} 4$ & $0(0)$ & $2(1)$ & & $0(0)$ & $2(1)$ & & $0(0)$ & $2(1)$ & \\
\hline \multirow[t]{4}{*}{ Grade } & 1 & $91(28)$ & $45(26)$ & 0.245 & $91(29)$ & $45(25)$ & 0.22 & $94(31)$ & $42(22)$ & 0.032 \\
\hline & 2 & $\begin{array}{l}200 \\
(62)\end{array}$ & $\begin{array}{l}105 \\
(61)\end{array}$ & & $18(6)$ & $\begin{array}{l}116 \\
(63)\end{array}$ & & $\begin{array}{l}184 \\
(60)\end{array}$ & $\begin{array}{l}121 \\
(64)\end{array}$ & \\
\hline & 3 & $26(8)$ & $15(9)$ & & $27(9)$ & $14(8)$ & & $24(8)$ & $17(9)$ & \\
\hline & 4 & $5(2)$ & $8(5)$ & & $5(2)$ & $8(4)$ & & $4(1)$ & $9(5)$ & \\
\hline $\begin{array}{l}\text { Tumour } \\
\text { diamater }\end{array}$ & $\mathrm{cm}$ & $\begin{array}{l}3.4 \\
(2.5- \\
5.0)\end{array}$ & $\begin{array}{l}4(2.5- \\
6.0)\end{array}$ & 0.071 & $\begin{array}{l}3.45 \\
(2.4- \\
5.0)\end{array}$ & $\begin{array}{l}4(2.5- \\
6.5)\end{array}$ & 0.026 & $\begin{array}{l}3.4 \\
(2.1- \\
4.5)\end{array}$ & $4(3-7)$ & $\begin{array}{l}< \\
0.0001\end{array}$ \\
\hline \multirow[t]{2}{*}{$\begin{array}{l}\text { Histology } \\
\text { type }\end{array}$} & $\begin{array}{l}\text { clear } \\
\text { cell }\end{array}$ & $\begin{array}{l}257 \\
(80)\end{array}$ & $\begin{array}{l}138 \\
(80)\end{array}$ & 0.127 & $\begin{array}{l}248 \\
(79)\end{array}$ & 147 & 0.9 & $\begin{array}{l}245 \\
(80)\end{array}$ & $\begin{array}{l}150 \\
(79)\end{array}$ & 0.9 \\
\hline & other & $65(20)$ & $35(20)$ & & $64(21)$ & $36(20)$ & & $61(20)$ & $39(21)$ & \\
\hline \multirow[t]{2}{*}{ Surgery } & $\mathrm{RN}$ & $91(28)$ & $65(38)$ & 0.042 & $85(27)$ & 71 (39) & 0.009 & $77(25)$ & $79(42)$ & 0.0001 \\
\hline & NSS & $\begin{array}{l}231 \\
(72)\end{array}$ & $\begin{array}{l}108 \\
(62)\end{array}$ & & $\begin{array}{l}227 \\
(73)\end{array}$ & $\begin{array}{l}112 \\
(61)\end{array}$ & & $\begin{array}{l}229 \\
(75)\end{array}$ & $\begin{array}{l}110 \\
(58)\end{array}$ & \\
\hline \multicolumn{11}{|l|}{ Outcomes } \\
\hline \multirow[t]{2}{*}{ Recurrence } & No & $\begin{array}{l}290 \\
(90)\end{array}$ & $\begin{array}{l}152 \\
(88)\end{array}$ & 0.45 & $\begin{array}{l}285 \\
(91)\end{array}$ & $\begin{array}{l}157 \\
(86)\end{array}$ & 0.07 & $\begin{array}{l}280 \\
(92)\end{array}$ & $\begin{array}{l}162 \\
(86)\end{array}$ & 0.052 \\
\hline & Yes & $32(10)$ & $21(12)$ & & $27(9)$ & $26(14)$ & & $26(8)$ & $27(14)$ & \\
\hline
\end{tabular}




\begin{tabular}{|c|c|c|c|c|c|c|c|c|c|c|}
\hline & & $\begin{array}{l}\text { NLR< } \\
2.75\end{array}$ & $\begin{array}{l}\text { NLR } \geq \\
2.76\end{array}$ & & $\begin{array}{l}\text { SIRI } \leq \\
2.25\end{array}$ & $\begin{array}{l}\text { SIRI> } \\
2.25\end{array}$ & & $\begin{array}{l}\text { SII } \leq \\
628\end{array}$ & $\begin{array}{l}\mathrm{S} \| 1> \\
628\end{array}$ & \\
\hline \multirow[t]{2}{*}{ Death } & No & $\begin{array}{l}299 \\
(93)\end{array}$ & $\begin{array}{l}153 \\
(88)\end{array}$ & 0.13 & $\begin{array}{l}292 \\
(94)\end{array}$ & $\begin{array}{l}160 \\
(87)\end{array}$ & 0.021 & $\begin{array}{l}286 \\
(93)\end{array}$ & $\begin{array}{l}166 \\
(88)\end{array}$ & 0.034 \\
\hline & Yes & $23(7)$ & $20(12)$ & & $20(6)$ & $23(13)$ & & $20(7)$ & $23(12)$ & \\
\hline \multirow[t]{2}{*}{$\begin{array}{l}\text { Cancer } \\
\text { Death }\end{array}$} & No & $\begin{array}{l}314 \\
(98)\end{array}$ & $\begin{array}{l}158 \\
(91)\end{array}$ & 0.003 & $\begin{array}{l}305 \\
(98)\end{array}$ & $\begin{array}{l}167 \\
(91)\end{array}$ & 0.002 & $\begin{array}{l}300 \\
(98)\end{array}$ & $\begin{array}{l}172 \\
(91)\end{array}$ & 0.0006 \\
\hline & Yes & 8 (2) & $15(9)$ & & $7(2)$ & $16(9)$ & & $6(2)$ & $17(9)$ & \\
\hline
\end{tabular}

Cut-off values for SIRI, SII, NLR, PLR, LMR, dNLR, HPR and NER were calculated to predict cancer-specific survival using ROC. For comparison different ROCs with respective areas under the curve (AUC) were demonstrated (Fig. 1).

\section{Systemic inflammatory markers predict CSS in Kaplan-Meier analysis}

When analyzing the preoperative systemic inflammatory markers, the CSS was worse in patients presenting with elevated NLR ( $\geq 2.76$ vs. $<2.76, p=0.0005)$, dNLR ( $>1.8$ vs. $\leq 1.8, p=0.012)$, PLR ( $>170$ vs. $\leq 170, p=0.0189)$, NER ( $>$ 0.95 vs. $\leq 0.95, p=0.0078)$, SIRI (>2.25 vs. $\leq 2.25, p=0.0005)$, and SII (>628vs. $\leq 628, p<0.0001)$, and lower HPR ( $\leq$ 0.53 vs. $>0.53, p=0.03$ ) and LMR values ( $<2.0$ vs. $\geq 2.0, p=0.01)$ (Fig. 2$)$.

\section{Univariate and multivariate analysis of CSS}

Univariate analysis of the associations between CSS and clinicopathologic factors revealed that age $(p=0.009)$, tumour grade $(p<0.0001)$, staging $(p<0.0001)$, diameter of tumor $(p<0.0001)$ but not the histological type of RCC $(p=0.55)$ were found to be significant predictors (Table 3$)$. As for hematological markers, higher SIRI $(p=0.001)$, SII $(p=0.0004)$, $\operatorname{NLR}(p=0.001), \operatorname{NER}(p=0.01), \operatorname{PLR}(0.02), \operatorname{dNLR}(p=0.02)$ and lower LMR $(p=0.015)$ and HPR $(p=0.04)$ values were associated with worse CSS. 
Table 3

Univariate analysis for CSS in patients with RCC treated with partial or radical nephrectomy with respective values of the hematological markers

\begin{tabular}{|c|c|c|c|c|c|}
\hline \multicolumn{6}{|c|}{ Univariate analysis- whole cohort- CSS } \\
\hline Clinicopathological features & & HR & Lower $95 \% \mathrm{Cl}$ & Upper $95 \% \mathrm{Cl}$ & $\mathbf{p}$ \\
\hline Age & (years) & 1.05 & 1.01 & 1.09 & 0.009 \\
\hline Gender & male vs female & 0.67 & 0.30 & 1.52 & 0.33 \\
\hline \multirow[t]{4}{*}{ Grade } & ref $=$ Grade 1 & 1 & & & $<.0001$ \\
\hline & Grade 2 & 3.121 & 0.698 & 13.889 & 0.13 \\
\hline & Grade 3 & 6.389 & 1.065 & 38.462 & 0.042 \\
\hline & Grade 4 & 90.31 & 17.544 & 500 & $<.0001$ \\
\hline \multirow[t]{6}{*}{ Staging } & ref = stage $\mathrm{T} 1 \mathrm{a}$ & 1 & - & - & $<0.0001$ \\
\hline & stage $T 1 b$ & 6.79 & 1.7 & 27.03 & 0.007 \\
\hline & stage $\mathrm{T} 2 \mathrm{a}$ & 16.83 & 3.4 & 83.33 & 0.001 \\
\hline & stage $T 2 b$ & 23.71 & 3.97 & 142.86 & 0.001 \\
\hline & stage T3 & 21.52 & 5.56 & 83.33 & $<0.0001$ \\
\hline & stage $\mathrm{T} 4$ & 345.13 & 55.56 & - & $<0.0001$ \\
\hline Tumour diameter & & 1.27 & 1.16 & 1.40 & $<0.0001$ \\
\hline Type of RCC & clear cell vs other & 1.45 & 0.43 & 4.89 & 0.55 \\
\hline \multicolumn{6}{|l|}{ Hematological markers } \\
\hline SIRI & high vs low & 4.24 & 1.75 & 10.32 & 0.001 \\
\hline SII & high vs low & 5.33 & 2.10 & 13.54 & 0.0004 \\
\hline NLR & high vs low & 4.11 & 1.74 & 9.70 & 0.001 \\
\hline NER & high vs low & 3.33 & 1.30 & 8.38 & 0.01 \\
\hline PLR & high vs low & 2.63 & 1.14 & 6.08 & 0.02 \\
\hline HPR & low vs high & 2.382 & 1.050 & 5.40 & 0.04 \\
\hline dNLR & high vs low & 2.79 & 1.21 & 6.46 & 0.02 \\
\hline LMR & low vs high & 2.84 & 1.23 & 6.56 & 0.015 \\
\hline
\end{tabular}

In multivariate analysis, staging $(p<0.0001)$, grade $(p=0.001)$, age $(p=0.02)$ and high SIRI $(p=0.015)$ (alternatively high SII $(p=0.02)$ and high NLR $(p=0.02)$ ) were significant predictors of CSS with eminent accuracy $(C$-index 0.903$)$ (Table 4). As inflammatory markers demonstrated a strong correlation between each other (SII vs. SIRI $r=0.76, p<$ 0.0001 ; SIRI vs. NLR $r=0.80, p<0.0001$; NLR vs. SII $r=0.89 p<0.0001)$, we decided to create three alternative models using a single systemic inflammatory marker for each model. Model without the inclusion of any inflammatory marker was characterized by a slightly lower C-index (C-index 0.882). 
Table 4

Multivariate analyses for CSS in patients with RCC treated with partial or radical nephrectomy with respective values of the hematological markers Note: hematologic markers were included in separate models, three models were developed with the following accuracy- C-index: *0.903, $\star * 0.902, * \star * 0.890$. Model without markers achieved C-index $=0.877$.

\begin{tabular}{|c|c|c|c|c|c|}
\hline \multicolumn{6}{|c|}{ Multivariate analysis- whole cohort- CSS } \\
\hline Predictive factor & & HR & Lower $95 \% \mathrm{Cl}$ & Upper 95\% Cl & $p$ \\
\hline \multirow[t]{6}{*}{ Staging } & Ref = stage $\mathrm{T} 1 \mathrm{a}$ & 1 & & & $<0.0001$ \\
\hline & stage $\mathrm{T} 1 \mathrm{~b}$ & 5.16 & 1.28 & 20.83 & 0.02 \\
\hline & stage $\mathrm{T} 2 \mathrm{a}$ & 13.18 & 2.43 & 71.43 & 0.003 \\
\hline & stage $\mathrm{T} 2 \mathrm{~b}$ & 4.30 & 0.53 & 35.71 & 0.2 \\
\hline & stage T3 & 6.53 & 1.43 & 30.3 & 0.02 \\
\hline & stage T4 & 17.09 & 1.51 & 200 & 0.02 \\
\hline \multirow[t]{4}{*}{ Grade } & ref = Grade 1 & 1 & & & 0.001 \\
\hline & Grade 2 & 1.630 & 0.347 & 7.634 & 0.53 \\
\hline & Grade 3 & 1.542 & 0.218 & 10.87 & 0.66 \\
\hline & Grade 4 & 25.837 & 3.367 & 200 & 0.002 \\
\hline Age & (years) & 3.512 & 1.272 & 9.701 & 0.02 \\
\hline $\mathrm{SIRI}$ * & high vs low & 3.154 & 1.174 & 8.472 & 0.013 \\
\hline SII** & high vs low & 3.30 & 1.21 & 8.99 & 0.02 \\
\hline$N L R^{\star \star \star}$ & high vs low & 2.616 & 1.006 & 6.802 & 0.043 \\
\hline
\end{tabular}

\section{Systemic inflammatory markers predict OS in Kaplan-Meier analysis}

Overall survival was significantly shorter in individuals with high NLR ( $\geq 2.76$ vs. $<2.76, p=0.027)$, PLR $(>170$ vs. $\leq 170$, $p=0.027)$, NER (>0.95 vs. $\leq 0.95, p=0.0016)$, SIRI (>2.25 vs. $\leq 2.25, p=0.0098)$, SII (>628 vs. $\leq 628, p<0.008)$, and lower LMR (<2.0 vs. $\geq 2.0, p=0.03$ ) (Fig. 3). HPR and dNLR values were not significantly associated with OS.

\section{Univariate and multivariate analysis of OS}

Associations between OS and selected clinicopathological variables or laboratory parameters are shown in Tables 5 and 6. In the univariate analysis age $(p=0.004)$, tumour grade $(p<0.0001)$, staging $(p<0.0001)$, diameter of tumor $(p=$ $0.001)$, but not type of RCC ( $p=0.15)$ were proved to be significant prognostic factors (Table 5). Results of peripheral blood counts reveled that higher SIRI $(p=0.01)$, SII $(p=0.009), \operatorname{NLR}(p=0.03)$, NER $(p=0.0025)$, PLR $(0.03)$, and lower $\operatorname{LMR}(p=0.03)$ values were associated with OS. 
Table 5

Univariate analysis for OS in patients with RCC treated with partial or radical nephrectomy with respective values of the hematological markers

\begin{tabular}{|c|c|c|c|c|c|}
\hline \multicolumn{6}{|c|}{ Univariate analysis- whole cohort-OS } \\
\hline Clinicopathological features & & HR & Lower $95 \% \mathrm{Cl}$ & Upper 95\% Cl & $\mathbf{p}$ \\
\hline Age & (years) & 1.040 & 1.013 & 1.067 & 0.004 \\
\hline Gender & male vs female & 1.404 & 0.732 & 2.693 & 0.30 \\
\hline \multirow[t]{4}{*}{ Grade } & ref $=$ Grade 1 & 1 & & & $<0.0001$ \\
\hline & Grade 2 & 2.818 & 1.085 & 7.299 & 0.03 \\
\hline & Grade 3 & 4.293 & 1.241 & 14.925 & 0.02 \\
\hline & Grade 4 & 36.813 & 10.989 & 125 & $<.0001$ \\
\hline \multirow[t]{6}{*}{ Staging } & ref $=$ stage $T 1 a$ & 1 & & & $<0.0001$ \\
\hline & stage $\mathrm{T} 1 \mathrm{~b}$ & 1.61 & 0.73 & 3.55 & 0.24 \\
\hline & stage $\mathrm{T} 2 \mathrm{a}$ & 3.61 & 1.23 & 10.64 & 0.02 \\
\hline & stage $T 2 b$ & 3.73 & 0.87 & 16.13 & 0.076 \\
\hline & stage T3 & 3.42 & 1.44 & 8.13 & 0.005 \\
\hline & stage T4 & 62.5 & 13.89 & 250 & $<0.0001$ \\
\hline Tumour diameter & & 1.149 & 1.055 & 1.250 & 0.001 \\
\hline Type of RCC & clear cell vs other & 2.099 & 0.750 & 5.876 & 0.15 \\
\hline \multicolumn{6}{|l|}{ Hematological markers } \\
\hline SIRI & high vs low & 2.161 & 1.186 & 3.936 & 0.01 \\
\hline SII & high vs low & 2.206 & 1.210 & 4.022 & 0.009 \\
\hline NLR & high vs low & 1.946 & 1.067 & 3.548 & 0.03 \\
\hline NER & high vs low & 2.735 & 1.426 & 5.247 & 0.0025 \\
\hline PLR & high vs low & 2.027 & 1.070 & 3.839 & 0.03 \\
\hline HPR & low vs high & 1.761 & 0.964 & 3.217 & 0.06 \\
\hline $\mathrm{dNLR}$ & high vs low & 1.587 & 0.870 & 2.892 & 0.13 \\
\hline LMR & low vs high & 1.915 & 1.053 & 3.482 & 0.03 \\
\hline
\end{tabular}


Table 6

Multivariate analysis for OS in patients with RCC treated with partial or radical nephrectomy with respective values of the hematological markers Note: hematologic markers were included in separate models, three models were developed with the following accuracy- C-index: ${ }^{\star} 0.750, \star \star 0.747, * \star \star 0.745$. Model without markers achieved C-index $=$ 0.725 .

\begin{tabular}{|llllll|}
\hline \multicolumn{4}{|l|}{ Multivariate analysis- whole cohort-OS } & & \\
\hline Predictive factor & & HR & Lower 95\% Cl & Upper 95\% Cl & P \\
\hline Grade & ref = Grade 1 & 1 & & & $<.0001$ \\
\hline & Grade 2 & 2.733 & 1.05 & 7.09 & 0.04 \\
\hline & Grade 3 & 4.012 & 1.155 & 13.89 & 0.03 \\
\hline Age & Grade 4 & 34.011 & 10 & 111.11 & $<.0001$ \\
\hline NER* & (years) & 1.04 & 1.01 & 1.07 & 0.002 \\
\hline SIRI ** & high vs low & 2.395 & 1.236 & 4.643 & 0.01 \\
\hline SII*** & high vs low & 1.87 & 1.02 & 3.43 & 0.04 \\
\hline
\end{tabular}

In multivariate analysis, tumour grade $(p<.0001)$, age $(p=0.002)$ and high NER $(p=0.01)$ (high SII $(p=0.04)$ and high SIRI $(p=0.04)$ in alternative models) were significant predictors of OS with acceptable accuracy (C-indexes 0.75$)$ (Table 6).

\section{Systemic inflammatory markers predict RFS in Kaplan-Meier analysis}

The RFS was shorter in individuals with elevated PLR ( $>170$ vs. $\leq 170, p=0.0028)$, NER $(>0.95$ vs. $\leq 0.95, p=0.0016)$, SIRI (>2.25 vs. $\leq 2.25, p=0.018)$, SII (>628 vs. $\leq 628, p=0.01)$, and lower HPR values $(\leq 0.53$ vs. $>0.53, p=0.0046)$ (Fig. 4). NLR, dNLR, and LMR failed to prove to be significantly associated with RFS.

\section{Univariate and multivariate analysis of RFS}

In univariate analysis, the associations between RFS and pathologic factors, tumour grade $(p<0.0001)$, stage $(p<$ $0.0001)$, diameter of tumour ( $p<0.0001)$ were found to be significant (Table 7). Furthermore, higher SIRI $(p=0.02)$, SII $(p=0.02)$, PLR $(p=0.004)$, NER $(p=0.03)$ and lower HPR $(p=0.01)$ values were associated with RFS. 
Table 7

Univariate analysis for RFS in patients with RCC treated with partial or radical nephrectomy with respective values of the hematological markers

\begin{tabular}{|c|c|c|c|c|c|}
\hline \multicolumn{6}{|c|}{ Univariate analysis- whole cohort-RFS } \\
\hline Clinicopathological features & & $\mathrm{HR}$ & Lower $95 \% \mathrm{Cl}$ & Upper 95\% Cl & $\mathrm{p}$ \\
\hline Age & (years) & 1.008 & 0.986 & 1.030 & 0.45 \\
\hline Gender & male vs female & 0.705 & 0.411 & 1.210 & 0.20 \\
\hline \multirow[t]{4}{*}{ Grade } & ref $=$ Grade 1 & & & & $<.0001$ \\
\hline & Grade 2 & 2.132 & 0.934 & 4.878 & 0.07 \\
\hline & Grade 3 & 5.593 & 2.079 & 15.152 & 0.001 \\
\hline & Grade 4 & 30.950 & 10.989 & 90.909 & $<.0001$ \\
\hline \multirow[t]{6}{*}{ Staging } & ref $=$ stage $\mathrm{T} 1 \mathrm{a}$ & 1 & & & $<.0001$ \\
\hline & stage $\mathrm{T} 1 \mathrm{~b}$ & 1.86 & 3.88 & 0.89 & \\
\hline & stage $\mathrm{T} 2 \mathrm{a}$ & 6.41 & 15.15 & 2.7 & \\
\hline & stage $T 2 b$ & 6.41 & 21.74 & 1.9 & \\
\hline & stage T3 & 5.62 & 11.76 & 2.69 & \\
\hline & stage T4 & 19.23 & 142.86 & 2.55 & \\
\hline Tumour diameter & & 1.215 & 1.133 & 1.304 & $<.0001$ \\
\hline Type of RCC & clear cell vs other & 1.517 & 0.684 & 3.361 & 0.30 \\
\hline \multicolumn{6}{|l|}{ Hematological markers } \\
\hline SIRI & high vs low & 1.895 & 1.104 & 3.254 & 0.02 \\
\hline SII & high vs low & 1.946 & 1.133 & 3.343 & 0.02 \\
\hline NLR & high vs low & 1.392 & 0.801 & 2.419 & 0.24 \\
\hline NER & high vs low & 1.843 & 1.059 & 3.206 & 0.03 \\
\hline PLR & high vs low & 2.309 & 1.313 & 4.059 & 0.004 \\
\hline HPR & low vs high & 2.147 & 1.250 & 3.689 & 0.006 \\
\hline dNLR & high vs low & 1.328 & 0.771 & 2.288 & 0.30 \\
\hline LMR & low vs high & 1.484 & 0.862 & 2.556 & 0.15 \\
\hline
\end{tabular}

In multivariate analysis, only tumour grade $(p<0.0001)$ and tumor diameter $(p=0.009)$ were independent predictors of RFS (Table 8). 
Table 8

Multivariate analysis for RFS in patients with RCC treated with partial or radical nephrectomy with respective values of the hematological markers

\begin{tabular}{|llllll|}
\hline \multicolumn{7}{|l}{ Multivariate analysis- whole cohort-RFS } & & & \\
\hline Predictive factor & & HR & Lower 95\% Cl & Upper 95\% Cl & P \\
\hline Grade & ref = Grade 1 & 1 & & & $<0.0001$ \\
\hline & Grade 2 & 1.847 & 0.80 & 4.26 & 0.15 \\
& Grade 3 & 4.701 & 1.12 & 9.80 & 0.03 \\
\hline & Grade 4 & 23.92 & 5.46 & 52.63 & $<.0001$ \\
\hline Tumour diameter & $(\mathrm{cm})$ & 1.129 & 1.03 & 1.23 & 0.007 \\
\hline
\end{tabular}

\section{Discussion}

In this study, we evaluated the prognostic value of several systemic inflammatory markers in patients with localized or locally advanced renal cell carcinoma. We showed a comprehensive comparison of eight different blood count-derived markers (NLR, PLR, LMR, NER, HPR, SIRI SII), which all stratified RCC patients according to CSS.

There is a growing number of studies focusing on the involvement of inflammatory markers in the clinical practice of individuals that developed RCC $[4,6,7,9,14]$. The available papers, however, emphasize the role of particular parameters (e.g. NLR, PLR, LMR) derived from peripheral blood counts, but direct comparisons between different markers are lacking. Recently, novel inflammatory indices such as systemic SIRI and SII were also reported as prognostic for RCC oncological outcomes $[4,15]$. Furthermore, hematological parameters have a well-documented prognostic role in the setting of metastatic RCC, in which IMDC and MSKCC models are mainly used [16, 17].

In the present paper, we found that patients treated surgically for localized or locally advanced RCC, who presented preoperatively with higher NLR, PLR, SIRI, SII, NER, dNLR, and lower LMR and HPR, had a significantly worse CSS. Multivariate analysis showed that tumour stage, grade, age and inflammatory markers constituted independent factors predicting CSS. As the markers of systemic inflammatory response demonstrate a strong correlation between each other, we decided to create three alternative models using a single systemic inflammatory marker for each multivariate model. The model based on clinicopathologic features, which did not include any inflammatory markers was characterized by already high accuracy (C-index 0.877 ). Further addition of inflammatory indices provided more accurate prognostic information. The CSS predictive model with the highest accuracy included SIRI values and achieved a C-index of 0.903 , while alternative multivariate models included SII and NLR and were characterized by Cindex 0.902 and 0.890 , respectively. Therefore, different inflammatory markers seem to provide the same prognostic information, as they show a strong correlation with each other (SIRI, SII and NLR) and their incorporation into the model results in similar accuracy increment.

A variety of papers pinpoint the observation that preoperative blood count-derived parameters reflecting the systemic inflammatory response can help in the estimation of the prognosis in patients with RCC [14, 18-21]. Here, we focused on the role of the markers of the well-established usefulness (NLR, dNLR, PLR, LMR) in the survival analysis, together with the new tools that are easily calculated from peripheral blood count (SII and SIRI), and, finally, the implementation of red blood cells lineage derivatives (HPR and NER). NLR seems to be the most extensively studied marker shown to be predictive for survival in RCC patients [5]. A meta-analysis of 25 studies (24 retrospective, 1 prospective), including 6461 patients showed that pretreatment increased NLR is associated with worse OS in non-metastatic (localized) RCC (pooled HR $=1.90, p<0.001$ ) [22]. In patients treated surgically for RCC plenty of studies revealed the association 
between NLR and adverse pathologic tumour features (advanced staging, grading, depth of invasion, the presence of metastasis, tumour size [6, 7]). In our analysis, higher values of inflammatory markers (NLR, SIRI and SII) were associated with more advanced stage and radical nephrectomy as for the surgery type. Higher SIl was also related to higher grade and larger tumour diameter.

Cut-off values for the categorization of particular markers into elevated or decreased largely differ between studies. In cases of localized or locally advanced tumors, NLR above 3 was proven to be predictive of a higher risk of recurrence, shorter overall and progression-free survival and response to systemic treatment [23]. In the paper by Elghiaty et al. patients with non-metastatic RCC up to $7 \mathrm{~cm}$ in diameter, who presented with higher MLR (cut-off value of 0.21), were at greater risk of recurrence and had worse CSS [3]. Then, Hu et al. evaluated the prognostic significance of NLR (cut-off 2.78), dNLR (cut-off 2.05) and PLR (cut-off 185) in RCC cases treated with nephrectomy [6]. Firstly, there was an association between poorer OS and increased values of NLR, dNLR and PLR. Secondly, only NLR was found to be an independent risk factor for shorter survival. On the contrary, Lucca et al. enrolled localized RCC cases and described MLR as potentially the best prognostic tool (when compared to NLR and PLR) that reflects the systemic inflammatory response and enables the estimation of disease-free survival [19]. Conversely, another study proposed PLR as the most reliable marker for overall and cancer-specific survival prediction in patients with non-metastatic clear cell RCC, who underwent nephrectomy [24]. To sum up, conflicting reports on the superiority of different inflammatory markers exist and none could be clearly chosen as of highest prognostic value yet.

Here, we grouped the parameters in the analysis of survival, so as to determine their value in the prognosis of OS, CSS and RFS. In the present paper, the Kaplan-Meier curves revealed that higher NLR $(\geq 2.76)$, PLR $(>170)$, NER $(>0.95)$ and dNLR (> 1.8), and lower LMR (<2.0) and HPR ( $\leq 0.53)$ were associated with worse CSS, while only elevated NLR from the markers mentioned above was found to be an independent factor for CSS in multivariate analysis. Similarly, high NLR (but not dNLR) and PLR and lower LMR were associated with OS, but none of the above-mentioned, well-known markers constituted an independent predictor of OS. We also observed associations between increased PLR and worse RFS, while no influence was observed in the case of NLR, dNLR and LMR.

There is still limited evidence on the prognostic value of the novel markers of systemic inflammation such as systemic inflammatory response index and systemic immune-inflammation index [4, 15, 25]. Interestingly, in our study the novel markers of systemic inflammation i.e. higher SIRI and SII, occurred to be associated with worse CSS and represented independent predictors of CSS, comparably to NLR. CSS prognostic models including SIRI and SII outperformed the model that based on staging, grading and age alone. Moreover, in the multivariate analysis these inflammatory markers were also significant predictors of OS but did not prove to be an independent tool for RFS estimations. This observation complies with the paper by Chen et al., in which the authors reported worse CSS and OS in individuals with elevated SIRI [4]. Worth emphasizing is the fact that AUC for SIRI was far greater, when compared to NLR, PLR and MLR [4]. Furthermore, in our study SII exhibited superior AUC compared to other markers (including SIRI) for all investigated outcomes. Another retrospective study reported that elevated SII is associated with OS and CSS in non-metastatic RCC even after propensity score matching but the authors did not provide data on other inflammatory markers that could be obtained simultaneously [25]. A study by Ozbek et al. showed that high SIl is prognostic for worse overall, but not disease-specific survival in patients treated with nephrectomy due to RCC [15].

Some other researchers used the term SIRI for additional calculations, incorporating hemoglobin values [26, 27]. In the recent paper by Mao et al., one outlined similar results: SIRI proved to be an independent prognostic risk factor for OS and CSS with greater impact on prognostic nomograms when compared to LMR and hemoglobin [27]. In the current study, we also included hematological biomarkers derived from red blood cell lineage, i.e. HPR and NER. Among the potential mechanisms involved in the association between red blood cells and RCC, other authors listed e.g. anemia, systemic inflammation leading to iron deficiency or decrease in renal erythropoietin production [26]. Here, elevated NER 
was related to shorter recurrence-free, cancer-specific and overall survival in univariate analysis. Moreover, NER proved to be an independent risk factor for shorter overall survival, together with age and tumour grade in multivariate analysis. Decreased HPR was associated with poorer OS and CSS in univariate analysis, as well. To the best of our knowledge, the prognostic values of HPR and NER in RCC were hardly studied, and only few studies were performed that were focused on red blood cell derivatives $[28,29]$. Tang et al. described that low HPR $(<0.615)$ was associated with worse CSS, OS and PFS, but not RFS in bladder cancer patients [11]. To the best of our knowledge, neutrophil to red blood cell ratio (called NER in our study) has not been investigated in renal cell cancer, but was shown to be associated with worse disease-free survival and OS in breast cancer patients [30]. In our previous paper, we found that NER $>0.93$ was a predictor of poorer oncological outcomes (BCG failure) in high-risk non-muscle invasive bladder cancer [12].

None of the studied inflammatory markers were independently associated with recurrence-free survival. Although higher SIRI, SII, NER, PLR and lower HPR were related to shorter time to reccurence, only tumour diameter and grade served as independent predictors for RFS in the multivariable model. This corresponds with the majority of prognostic scores, which rely solely on histopathologic (staging, grading, necrosis, tumour diameter) and clinical features (age, symptoms) [31]. On the other hand, one of the meta-analysis reported the predictive value of elevated NLR for RFS/PFS in RCC (HR $=2.18$ ) [32]. Further studies investigating the role of inflammatory markers on the recurrence risk are warranted.

We are conscious of several limitations of our study that are listed below. It was a single-center retrospective analysis and selection bias could not be excluded. Some clinical data were not collected, and some other biomarkers easily obtained from peripheral blood count were not included e.g. lymphocyte to red blood cell ratio (LRR) [30]. Furthermore, no data on the postoperative values to be used in the follow-up were collected. Local and distant RCC recurrences were not analyzed separately. Nevertheless, we managed to analyse in the relatively large contemporary cohort the impact of several markers of the inflammatory response, easy to adopt in the patients with RCC for clinical purposes.

\section{Conclusions}

In our study, we compared the prognostic value provided by different inflammatory markers in patients with localized or locally advanced RCC. We have found that markers of systemic inflammation might provide additional prognostic value (especially SIRI, SII, NLR and NER), and further increase the predictive accuracy of available models. Clinicopathological features (tumour stage, grade and patient's age), however, remain the most important independent prognostic factors for oncological outcomes in RCC patients treated with nephrectomy. For the first time, we show the prognostic value of neutrophil-to-erythrocyte ratio, which constitutes an independent risk factor for overall survival.

\section{Abbreviations}

AUC - area under the curve

$\mathrm{Cl}$ - confidence interval

CSS - cancer-specific survival

dNLR - derived neutrophil/lymphocyte ratio

HPR - hemoglobin/platelet ratio

HR - hazard ratio

IQR - interquartile range

Page 16/22 
LMR - lymphocyte/monocyte ratio

LRR - lymphocyte to red blood cell ratio

MLR - monocyte/lymphocyte ratio

NER - neutrophil/erythrocyte ratio

NLR - neutrophil/lymphocyte ratio

OS - overall survival (OS)

PLR - platelet/lymphocyte ratio

RCC - renal cell carcinoma

RFS - recurrence-free survival

ROC - receiver operating curve

SII - systemic immune-inflammation index

SIRI - systemic inflammatory response index

\section{Declarations}

Ethics approval and consent to participate: The study was conducted under the Ethics committee vote AKBE/72/2021 of the Medical University of Warsaw. Informed consent was obtained from all subjects involved in the study.

Consent for publication: Not applicable

Availability of data and materials: The data presented in this study are available on reasonable request from the corresponding authors.

Competing interests: The authors declare that they have no competing interests

Funding: Publication of the study was founded by the Medical University of Warsaw.

Authors' Contributions: Conceptualization, Ł.Z. and A.Ś.; methodology, Ł.Z., and A.Ś.; software, A.Ś..; validation, Ł.Z., A.Ś., and P.Z.; formal analysis, Ł.Z., and A.Ś.; investigation, Ł.Z., A.Ś.; resources, Ł.Z., A.Ś., K.G., Ł.M., C.Ś., P.Z.; data curation, Ł.Z., and A.Ś.; writing-Ł.Z.; writing and re-editing- Ł.Z., A.Ś.; visualization, Ł.Z., A.Ś.; supervision, A.W., P.R.; project administration, Ł.Z., and A.Ś.; funding acquisition, Ł.Z. All authors have read and agreed to the published version of the manuscript.

Acknowledgments: Not applicable.

\section{References}

1. Agochukwu N, Shuch B. Clinical management of renal cell carcinoma with venous tumor thrombus. World J Urol. 2014;32:581-9. 
2. Kim SP, Weight CJ, Leibovich BC, Thompson RH, Costello BA, Cheville JC, et al. Outcomes and clinicopathologic variables associated with late recurrence after nephrectomy for localized renal cell carcinoma. Urology. 2011;78:1101-6.

3. Elghiaty A, Kim J, Jang WS, Park JS, Heo JE, Rha KH, et al. Predictive value of preoperative monocyte-lymphocyte ratio among patients with localized clear renal cell carcinoma of $</=7 \mathrm{~cm}$ on preoperative imaging. Med (Baltim). 2018;97:e13433.

4. Chen Z, Wang K, Lu H, Xue D, Fan M, Zhuang Q, et al. Systemic inflammation response index predicts prognosis in patients with clear cell renal cell carcinoma: a propensity score-matched analysis. Cancer Manag Res. 2019;11:909-19.

5. Chen DY, Uzzo RG, Viterbo R. Thinking beyond surgery in the management of renal cell carcinoma: the risk to die from renal cell carcinoma and competing risks of death. World J Urol. 2014;32:607-13.

6. Hu H, Yao X, Xie X, Wu X, Zheng C, Xia W, et al. Prognostic value of preoperative NLR, dNLR, PLR and CRP in surgical renal cell carcinoma patients. World J Urol. 2017;35:261-70.

7. Hutterer GC, Stoeckigt C, Stojakovic T, Jesche J, Eberhard K, Pummer K, et al. Low preoperative lymphocytemonocyte ratio (LMR) represents a potentially poor prognostic factor in nonmetastatic clear cell renal cell carcinoma. Urol Oncol. 2014;32:1041-8.

8. Colotta F, Allavena P, Sica A, Garlanda C, Mantovani A. Cancer-related inflammation, the seventh hallmark of cancer: links to genetic instability. Carcinogenesis. 2009;30:1073-81.

9. Fox P, Hudson M, Brown C, Lord S, Gebski V, De Souza P, et al. Markers of systemic inflammation predict survival in patients with advanced renal cell cancer. Br J Cancer. 2013;109:147-53.

10. Teishima J, Inoue S, Hayashi T, Mita K, Hasegawa Y, Kato M, et al. Impact of the systemic immune-inflammation index for the prediction of prognosis and modification of the risk model in patients with metastatic renal cell carcinoma treated with first-line tyrosine kinase inhibitors. Can Urol Assoc J. 2020;14:E582-E7.

11. Tang G, Zhen Y, Xie W, Wang Y, Chen F, Qin C, et al. Preoperative hemoglobin-platelet ratio can significantly predict progression and mortality outcomes in patients with T1G3 bladder cancer undergoing transurethral resection of bladder tumor. Oncotarget. 2018;9:18627-36.

12. Ślusarczyk A, Zapała P, Garbas K, Zapała $Ł$, Borkowski T, Radziszewski P. Blood count-derived inflammatory markers predict time to Bacillus Calmette-Guérin failure in high-risk non-muscle-invasive bladder cancer. Archives of Medical Science; 2020.

13. Shen J, Chen Z, Fan M, Lu H, Zhuang Q, He X. Prognostic value of pretreatment neutrophil count in metastatic renal cell carcinoma: a systematic review and meta-analysis. Cancer Manag Res. 2019;11:5365-74.

14. Otunctemur A, Dursun M, Besiroglu H, Ozer K, Horsanali O, Ozbek E. Clinical Significance of Preoperative Neutrophil - to - Lymphocyte Ratio in Renal Cell Carcinoma. Int Braz J Urol. 2016;42:678-84.

15. Ozbek E, Besiroglu H, Ozer K, Horsanali MO, Gorgel SN. Systemic immune inflammation index is a promising noninvasive marker for the prognosis of the patients with localized renal cell carcinoma. Int Urol Nephrol. 2020;52:1455-63.

16. Ramsey S, Lamb GW, Aitchison M, Graham J, McMillan DC. Evaluation of an inflammation-based prognostic score in patients with metastatic renal cancer. Cancer. 2007;109:205-12.

17. Bugdayci Basal F, Karacin C, Bilgetekin I, Oksuzoglu OB. Can Systemic Immune-Inflammation Index Create a New Perspective for the IMDC Scoring System in Patients with Metastatic Renal Cell Carcinoma? Urol Int. 2021;105:666-73.

18. Peyton CC, Abel EJ, Chipollini J, Boulware DC, Azizi M, Karam JA, et al. The Value of Neutrophil to Lymphocyte Ratio in Patients Undergoing Cytoreductive Nephrectomy with Thrombectomy. Eur Urol Focus. 2020;6:104-11. 
19. Lucca I, de Martino M, Hofbauer SL, Zamani N, Shariat SF, Klatte T. Comparison of the prognostic value of pretreatment measurements of systemic inflammatory response in patients undergoing curative resection of clear cell renal cell carcinoma. World J Urol. 2015;33:2045-52.

20. Rajwa P, Zyczkowski M, Paradysz A, Slabon-Turska M, Suliga K, Bujak K, et al. Novel hematological biomarkers predict survival in renal cell carcinoma patients treated with nephrectomy. Arch Med Sci. 2020;16:1062-71.

21. Qi Q, Zhuang L, Shen Y, Geng Y, Yu S, Chen H, et al. A novel systemic inflammation response index (SIRI) for predicting the survival of patients with pancreatic cancer after chemotherapy. Cancer. 2016;122:2158-67.

22. Shao Y, Wu B, Jia W, Zhang Z, Chen Q, Wang D. Prognostic value of pretreatment neutrophil-to-lymphocyte ratio in renal cell carcinoma: a systematic review and meta-analysis. BMC Urol. 2020;20:90.

23. Boissier R, Campagna J, Branger N, Karsenty G, Lechevallier E. The prognostic value of the neutrophil-lymphocyte ratio in renal oncology: A review. Urol Oncol. 2017;35:135-41.

24. Lee A, Lee HJ, Huang HH, Tay KJ, Lee LS, Sim SPA, et al. Prognostic Significance of Inflammation-associated Blood Cell Markers in Nonmetastatic Clear Cell Renal Cell Carcinoma. Clin Genitourin Cancer. 2020;18:304-13.

25. Hu X, Shao YX, Yang ZQ, Dou WC, Xiong SC, Li X. Preoperative systemic immune-inflammation index predicts prognosis of patients with non-metastatic renal cell carcinoma: a propensity score-matched analysis. Cancer Cell Int. 2020;20:222.

26. Gu L, Ma X, Wang L, Li H, Chen L, Li X, et al. Prognostic value of a systemic inflammatory response index in metastatic renal cell carcinoma and construction of a predictive model. Oncotarget. 2017;8:52094-103.

27. Mao W, Sun S, He T, Jin X, Wu J, Xu B, et al. Systemic Inflammation Response Index is an Independent Prognostic Indicator for Patients with Renal Cell Carcinoma Undergoing Laparoscopic Nephrectomy: A Multi-Institutional Cohort Study. Cancer Manag Res. 2021;13:6437-50.

28. Peng D, Zhang CJ, Tang Q, Zhang L, Yang KW, Yu XT, et al. Prognostic significance of the combination of preoperative hemoglobin and albumin levels and lymphocyte and platelet counts (HALP) in patients with renal cell carcinoma after nephrectomy. BMC Urol. 2018;18:20.

29. Yilmaz H, Yilmaz A, Demirag G. Prognostic significance of hemoglobin-to-red cell distribution width ratio in patients with metastatic renal cancer. Future Oncol 2021.

30. Wang Y, Wang H, Yin W, Lin Y, Zhou L, Sheng X, et al. Novel lymphocyte to red blood cell ratio (LRR), neutrophil to red blood cell ratio (NRR), monocyte to red blood cell ratio (MRR) as predictive and prognostic biomarkers for locally advanced breast cancer. Gland Surg. 2019;8:627-35.

31. Correa AF, Jegede O, Haas NB, Flaherty KT, Pins MR, Messing EM, et al. Predicting Renal Cancer Recurrence: Defining Limitations of Existing Prognostic Models With Prospective Trial-Based Validation. J Clin Oncol. 2019;37:2062-71.

32. Hu K, Lou L, Ye J, Zhang S. Prognostic role of the neutrophil-lymphocyte ratio in renal cell carcinoma: a metaanalysis. BMJ Open. 2015;5:e006404.

\section{Figures}



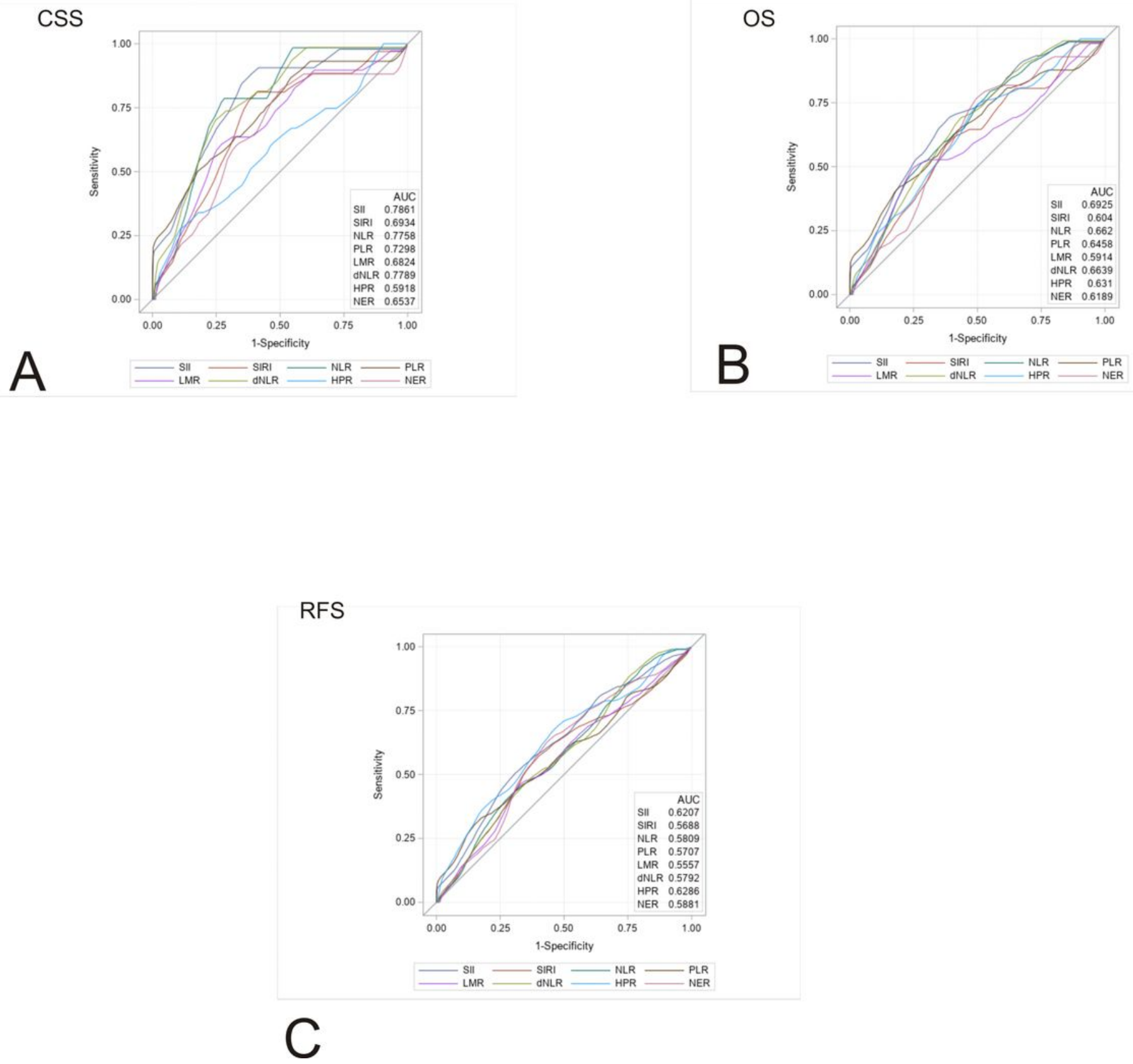

Figure 1

Receiver operating curves (ROC) for prediction of CSS (A), OS (B) and RFS (C) for hematological markers (NLR, dNLR, SIRI, PLR, LMR, HPR, NER) in the whole cohort (with respective AUC) in long-term analysis (42 months) 
NLR

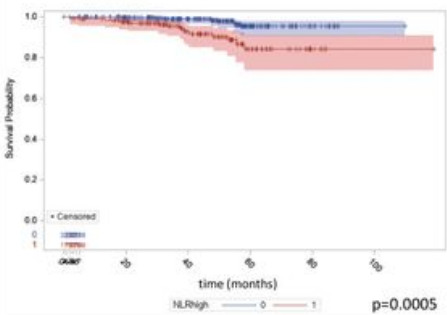

A

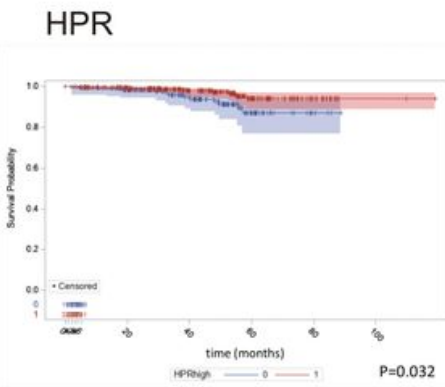

E

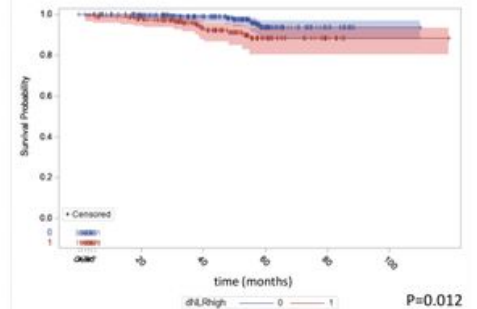

B

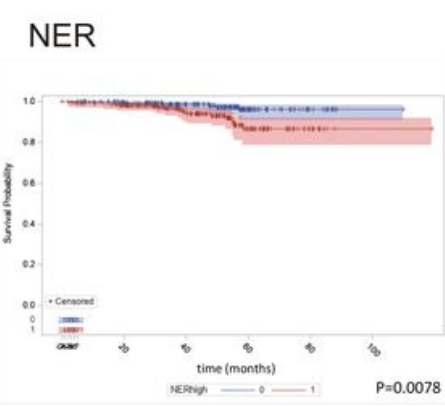

F
PLR

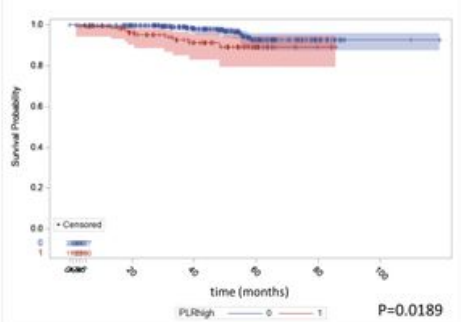

C

SIRI

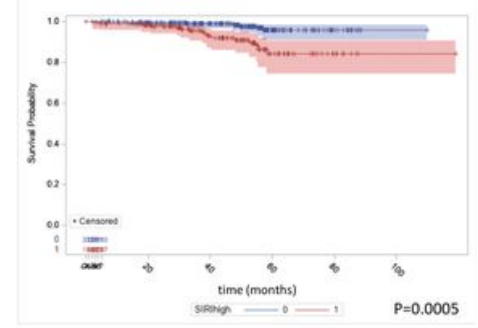

G
LMR

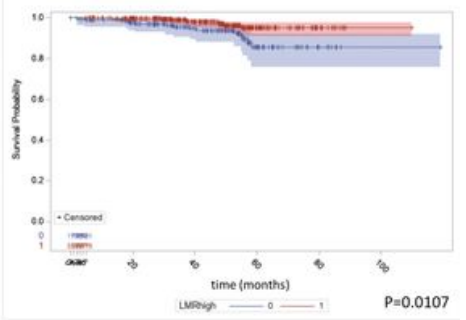

D

SII

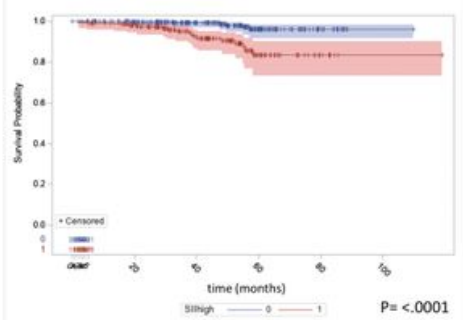

$\mathrm{H}$

Figure 2

The Kaplan-Meier curves for CSS in patients stratified by low vs. high NLR (A), dNLR (B), PLR (C), LMR (D), HPR (E), $\operatorname{NER}(\mathrm{F})$, SIRI (G) and SII (H)

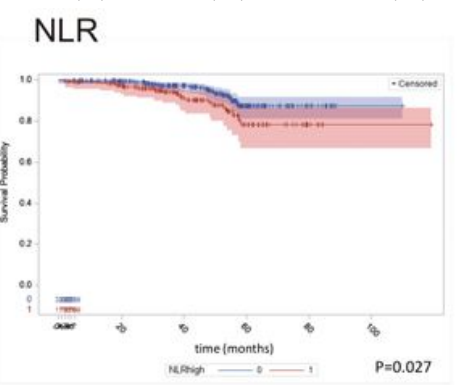

A

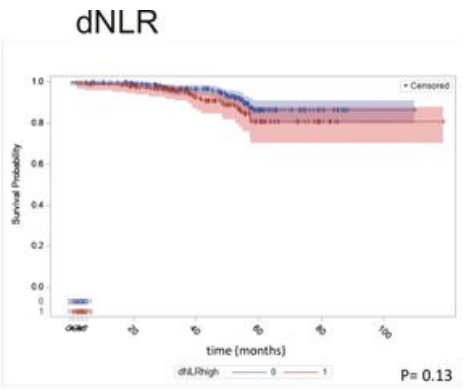

B

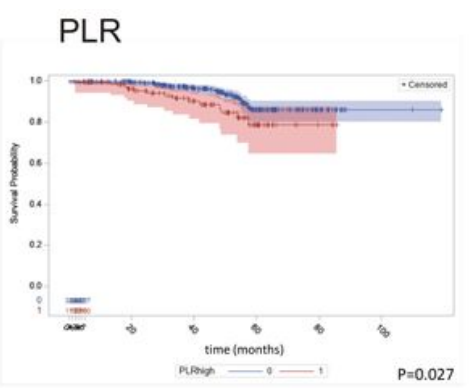

C

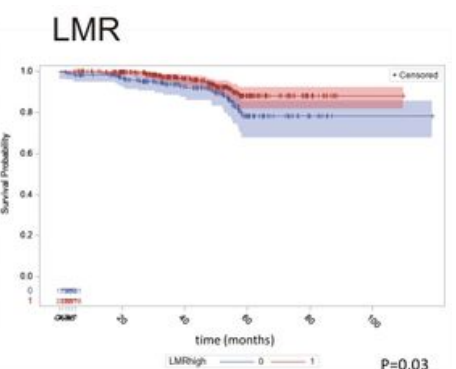

D
HPR

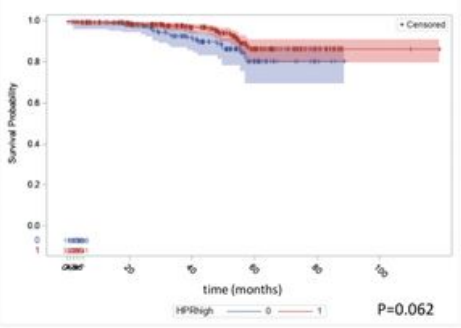

E
NER

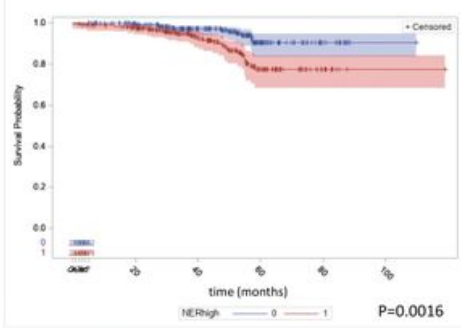

F

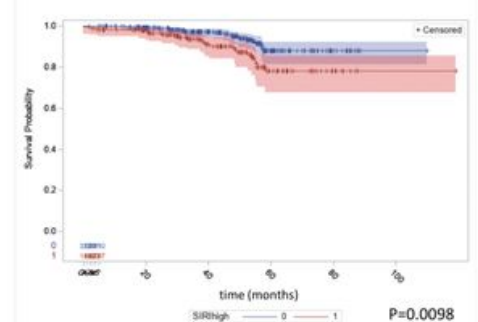

G
SII

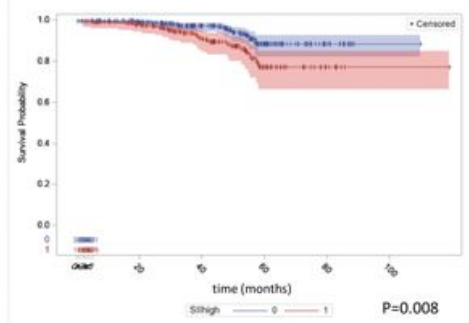

$\mathrm{H}$ 


\section{Figure 3}

The Kaplan-Meier curves for OS in patients stratified by low vs. high NLR (A), dNLR (B), PLR (C), LMR (D), HPR (E), NER (F), SIRI (G) and SII (H)
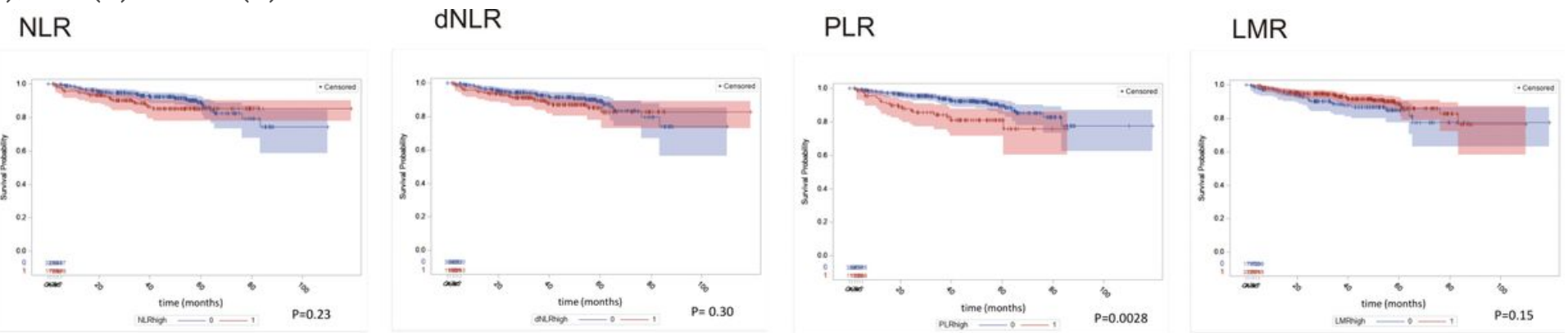
A

B

C

D

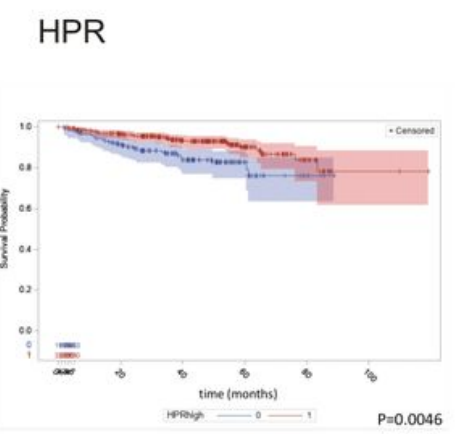

E
NER

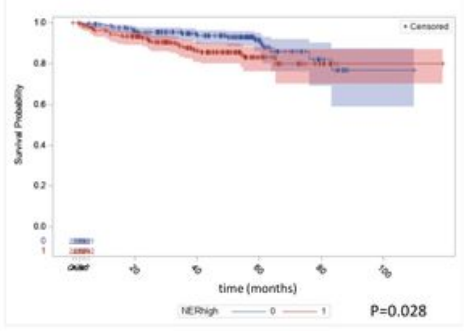

F
SIRI

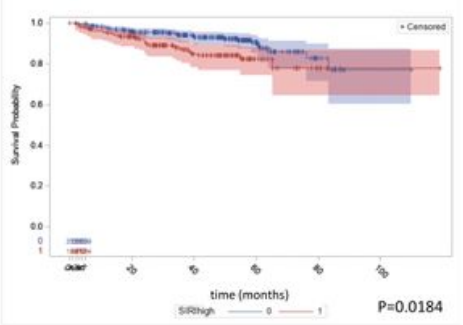

G

\section{SII}

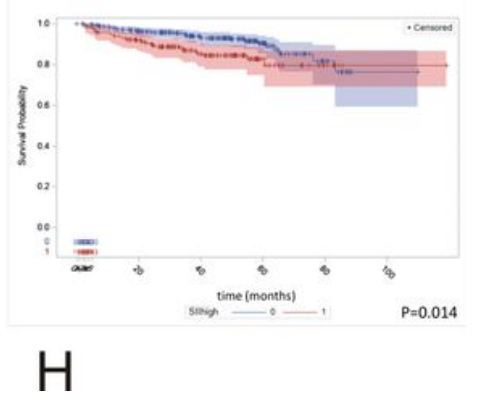

\section{Figure 4}

The Kaplan-Meier curves for RFS in patients stratified by low vs. high NLR (A), dNLR (B), PLR (C), LMR (D), HPR (E), $\operatorname{NER}(F), \operatorname{SIRI}(\mathrm{G})$ and SII (H) 